\title{
FLORÍSTICA DO CERRADO NA RESERVA BIOLÓGICA DE MOJI GUAÇU, SP.
}

\author{
Waldir Mantovani ${ }^{1}$ \\ Fernando Roberto Martins ${ }^{2}$
}

Recebido em 9-04-92. Aceito em 24-03-93

RESUMO - Foi analisada uma área de 343,42 ha situada em mancha disjunta do cerrado, no Município de Moji Guaçu (Reserva Biológica de Moji Guaçu), São Paulo, sudeste do Brasil $\left(22^{\circ} 15^{\prime}-16^{\prime} \mathrm{S}\right.$ e $\left.47^{\circ} 08^{\prime}-12^{\prime} \mathrm{W}\right)$. A área do cerrado estudada apresenta predomínio de fisionomias abertas, que vão do campo cerrado ao cerrado senso restrito. Foram amostradas as plantas vasculares em fase reprodutiva, em dezenove excursōes de coletga, com intervalos de 30-45 dias, quando eram anotados dados fenológicos e as formas de vida. Obtiveram-se 2019 exsicatas, distribuidos em 524 espécies, 286 gêneros e 82 famílias. Analisou-se a flora como um todo e os seus componentes herbáceo-subarbustivo e arbustivo-arbóreo, separadamente. Salientaram-se no componente arbustivo-arbóreo: Leguminosae, Myrtaceae e Rubiaceae e na flora como um todo e no seu componente campestre: Compositae, Gramineae e Leguminosae, que estão entre as famílias mais numerosas da flora mundial.

Palavras chave: Flora vascular, cerrado, Reserva Biológica de Moji Guaçu.

ABSTRACT - A 343.42 ha area of cerrado was analysed in a disjunct, marginal cerrado area in Moji Guaçu Municipality, São Paulo State (Moji Guaçu Biological Reserve, $22^{\circ} 15^{\prime}-16^{\prime} \mathrm{S}$ and $47^{\circ} 08^{\prime}-12 \mathrm{~W}$ ), southeastern Brazil. The predominantly open vegetation varies from "campo cerrado" to "cerrado" (restricted sense). The survey included all vascular plants in reproductive stage and was carried out in nineteen field trips to the area. A total of 2019 exsicata, representing 524 species, 286 genera and 83 families was collected. The cerrado flora as a whole and its two

1 - Depto Ecologia Geral - Instituto de Biocências, USP. Cx. P. 11.461. CEP 05499 - São Paulo, SP. Bolsista CNPq - Proc. 300.304/81

2 - Depto Morfologia e Sistemática Vegetais - Instituto de Biologia, UNICAP. Cx. P. 6.109. CEP 13081 - Campinas, SP. 
components (woody and herbaceous) were analysed separately. The wood flora is poorer than the herbaceous one, and is composed mainly of species belonging to Leguminosae, Myrtaceae and Rubiaceae. The cerrado flora as a whole, and the herbaceous component, in particular, are composed mainly of species belonging to Compositae, Gramineae and Leguminosae, three of the largest Angiosperm families.

Key words: vascular flora, cerrado, Moji Guaçu Biological Reserve

\section{Introdução}

O cerrado apresenta uma área nuclear (Labouriau, 1966), central (Rizzini, 1979) ou "core" (Eiten, 1972; Ferri, 1977) no Planalto Central Brasileiro, cobrindo os Estados de Goiás e de Tocantins e o Distrito Federal, a região oeste de Minas Gerais e da Bahia e o leste do Mato Grosso e do Mato Grosso do Sul (Figura 1). Esse núcleo ocupa um milhão e quinhentos mil quilômetros quadrados (Alvim \& Araujo, 1952) e é considerado o foco de irradiação da flora campestre (arbustivasubarbustiva) e da flora silvestre (matas secas e matas xeromórficas ou cerradões) (Rizzini, 1963b). Expande-se ininterruptamente em direção ao litoral do Maranhão e do Piauí, para a região sul de Rondônia, passando pelo centro-oeste do Mato Grosso e para o sudoeste do Mato Grosso do Sul, atingindo o leste do Paraguai (Eiten, 1972).

A flora do cerrado aparece, ainda que com um número variável de espécies componentes da sua flora nuclear, em áreas disjuntas: no norte, no Estado do Amazonas (Ducke \& Black, 1954), nos Territórios do Amapá (Azevedo, 1967) e de Roraima (Rodrigues, 1971; Takeuchi, 1960); no nordeste, nos Estados de Alagoas, Bahia, Ceará, Paraíba e Pernambuco (Rizzini, 1979; Veloso, 1964); e no sul, nos Estados de São Paulo (Borgonovi \& Chiarini, 1965; Eiten, 1970; Loefgren, 1896; Troppmair, 1974) e Paraná (Ferri, 1960; Stellfeld, 1950).

Com tal extensão, o cerrado apresenta uma flora muito rica e variável (Veloso, 1963), para a qual quase inexistem estudos (Ferri, 1978). A maioria dos trabalhos que abordam aspectos florísticos traz análises efetuadas a partir de levantamentos fitossociológicos, em geral restritos ao componente arbustivoarbóreo do cerrado.

Levantamentos florísticos, intensos e sistemáticos, foram feitos no cerrado na Lagoa Santa, MG (Warming, 1892), em Brasília (Ratter, 1980) e no Triângulo Mineiro (Goodland, 1969). Análises florísticas foram feitas por Goodland (1969), Rizzini (1963a) e por Warming (1892). Indicações e análises das relações entre os componentes do cerrado com outras formaçōes, florestais ou campestres, foram efetuadas por Heringer et al. (1977), por Rizzini (1963b) e por Warming (1892), entre outros. Neste trabalho espera-se contribuir para o conhecimento da flora do cerrado. 


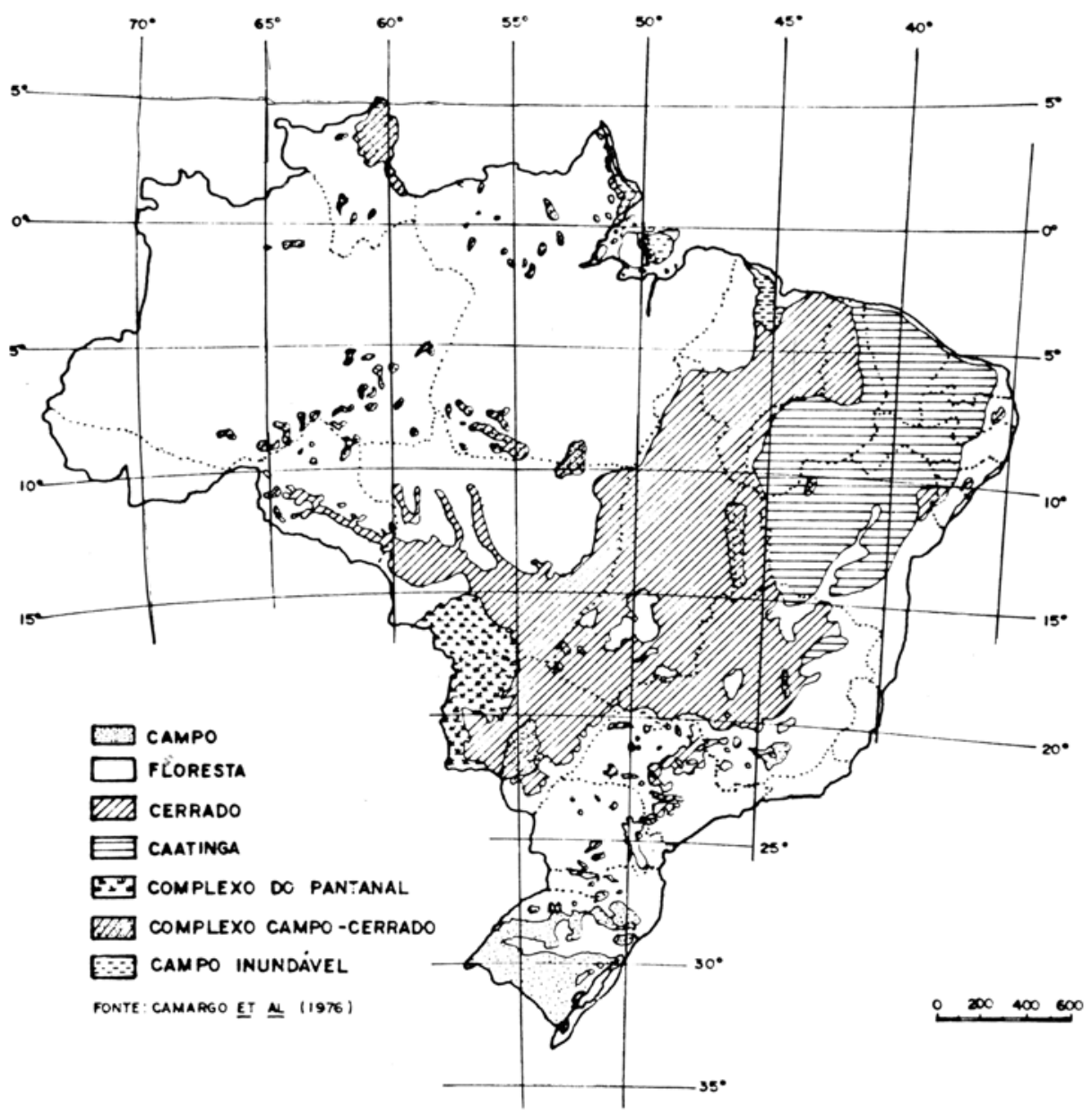

Figura 1 - Distribuição das formaçōes vegetais brasileiras.

\section{Material e Métodos}

A Reserva Biológica de Moji Guaçu é composta por duas áreas distintas (De Vuono et al., 1982), denominando-se de Reserva, neste trabalho, a gleba "A", com cerca de 343,42 ha. Está situada entre $22^{\circ} 15^{\prime}-16^{\prime} \mathrm{S}$ e $47^{\circ} 08^{\prime}-12^{\prime} \mathrm{W}$, no Município de Moji Guaçu, Estado de São Paulo, sob a responsabilidade administrativa do Instituto de Botânica da Secretaria do Meio Ambiente do Estado de São Paulo.

Situa-se em região sob o clima Cwa, de Koeppen, tendo sido bem caracterizada quanto ao clima nos trabalhos de De Vuono et al. (1986) e de Mantovani 
(1983). Os solos da Reserva foram analisados por Donzelli et al. (inédito) e por Perez $F^{2}$ et al. (1980), que os classificaram como solos da planície de inundação e Latossolos Vermelho-Amarelo, álico, A moderado, textura média (Unidade Laranja Azeda) e Vermelho-Amarelo, álico, A moderado, textura argilosa, relevo aplainado ou suave ondulado (Unidade Mato Dentro). A relação entre os solos e a vegetação foi detalhadamente analisada por Batista (1988).

A vegetação da Reserva foi estudada com ênfases diversas por diferentes autores (Batista, 1982; 1988; Eiten, 1963; Mantovani et al., 1985; Mantovani, 1987). É composta por floresta de galeria, campos úmidos e, na sua maior parte, pelo cerrado. A área, antes da ocupação por fazenda de gado bovino, era coberta por cerrado denso, semelhante ao que se observa na sua extremidade sudeste. Até 1964 a área da Reserva era pastejada e antes de 1960 sofria queimadas freqüentes nas partes, atualmente, com fisionomias mais abertas; a última queimada intensa ocorreu em julho de 1975, em trecho limitado.

Geadas fortes ocorreram em 1975, 1979 e 1981 e uma geada moderada atingiu a Reserva em 1980 (Mantovani, 1983), tendo sido mais atingidas as áreas de baixada, que correspondem à parte noroeste. Excetuando-se a parte do cerrado da região sudeste, todas as outras fisionomias sofreram cortes seletivos de espécimes.

As queimadas localizadas, as diferentes exposições às geadas, a drenagem do solo e os efeitos antropogênicos foram apontados por Gibbs et al. (1983) como os responsáveis pela existência de diversas fisionomias do cerrado na Reserva. Mantovani (1987) identificou 5 fisionomias: cerrado senso restrito, cerrado senso restrito de Rapanea, transição, campo cerrado e campo cerrado queimado (Figura 2), com base em fotos aéreas e nas excursōes de coletas

O levantamento florístico foi efetuado de outubro de 1979 a agosto de 1981, através de 19 excursões de coleta, com duração de uma semana, em intervalos de 30 a 45 dias. Os espécimes em fase florífera e/ou frutífera ou de formação de esporos, eram coletados em caminhadas efetuadas no sentido NW-SE da Reserva, acondicionados em sacos plásticos e prensados, posteriormente. Evitaram-se coletas nas matas e nos campos úmidos, procurando não amostrar espécimes atípicas, e numa faixa de $30-40 \mathrm{~m}$ de distância de cada margem da área do cerrado, tentando-se evitar a coleta de plantas invasoras.

As espécies foram incluídas em famílias de acordo com o sistema de Engler \& Prantl, indicado por Joly (1977), tendo sido identificadas através de bibliografia pertinente ou de comparaçōes com exsicatas depositadas no Herbário do Instituto de Botânica de São Paulo.

A relação de espécies a ser apresentada obecederá à ordem alfabética de famílias, gêneros e espécies, dentro dos grupos das Pteridophyta, Angiospermae Monocotyledoneae e Dicotyledoneae e trará indicaçōes acerca da forma de vida (Raunkiaer, 1934, adaptado por Mueller-Dombois \& Ellenberg, 1974), período de floração e da síndrome de dispersão dos diásporos de cada espécie (Pijl, 1972). 
Florística do cerrado...

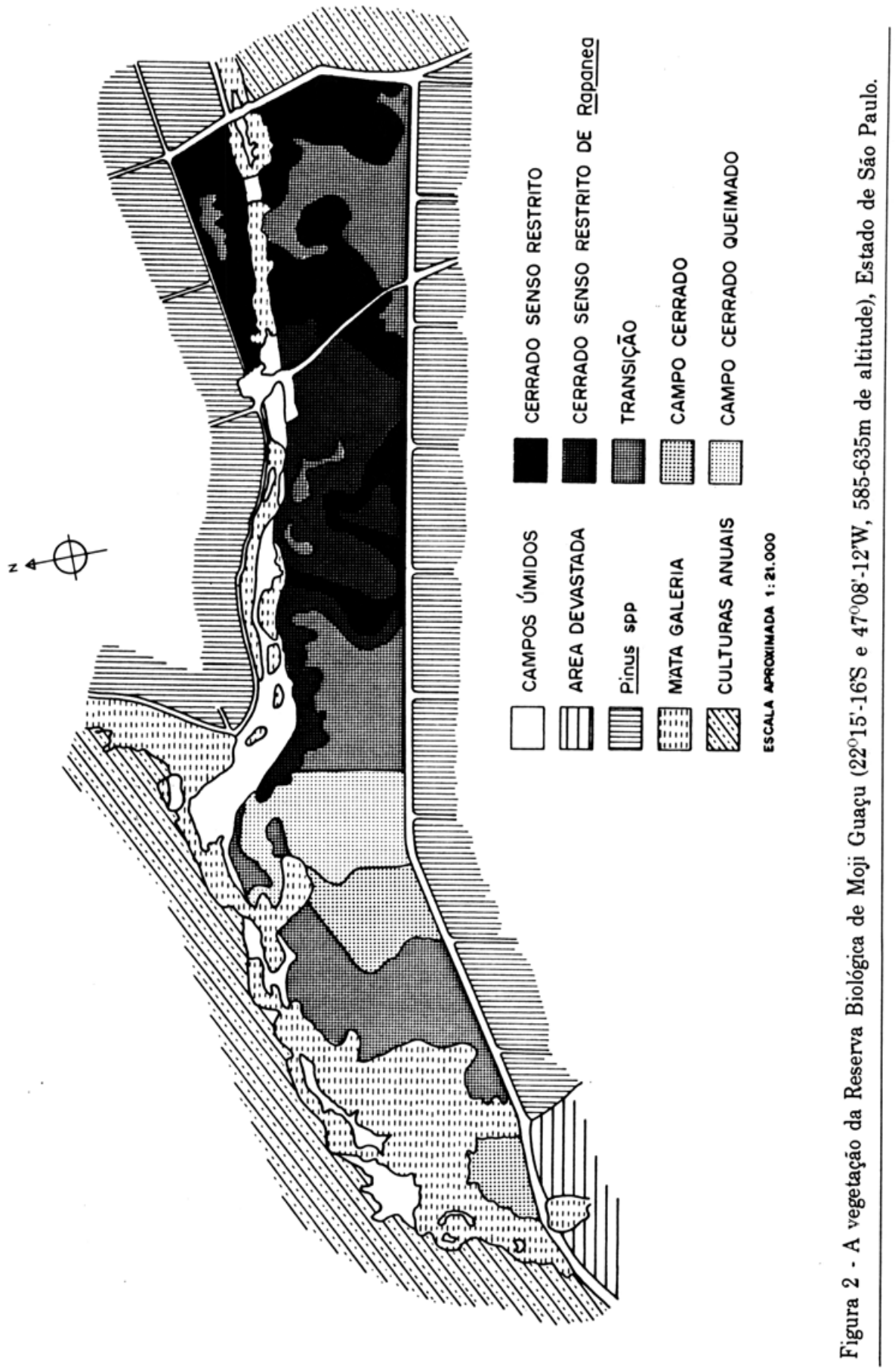




\section{Resultados e Discussão}

A flora obtida é apresentada na (Tabela 1). A coleta em caminhadas efetuadas a cada 30 a 45 dias, permitiu uma amostragem satisfatória da flora do cerrado da Reserva, ainda que muitas espécies herbáceas apresentem um ciclo epigeo de poucas semanas. Embora seja uma avaliação subjetiva, supōe-se que $70 \%$ a $90 \%$ das espécies daquela flora tenham sido amostrados.

A prensagem do material após o dia de coleta, acarretou boas exsicatas para a maioria das espécies, entretanto, para algumas famílias, como Euphorbiaceae, Convolvulaceae, Leguminosae-Mimosoideae, Malvaceae e Commelinaceae, recomenda-se a prensagem no local da coleta, visto serem muito sensíveis à perda de água ou apresentarem sensibilidade ao toque (Leguminosae-Mimosoideae).

Aproximadamente um terço das famílias encontradas na flora em estudo, ou $31,70 \%$ (vinte e cinco famílias), apresenta uma única espécie. Mais da metade dessas famílias, ou $58,42 \%$ (quarenta e oito delas), tem até três espécies e contém $15,43 \%$ do total encontrado (oitenta e uma espécies), como se observa na (Figura 3). As famílias que contêm o maior número de espécies são: Compositae, Leguminosae, Gramineae, Rubiaceae, Euphorbiaceae, Apocynaceae, Bignoniaceae, Cyperaceae, Malpighiaceae, Myrtaceae, Labiatae, Melastomataceae e Verbenaceae, respectivamente (Figura 4).

Comparando-se este trabalho com os de Goodland (1969), Ratter (1980) e de Warming (1892), o número de famílias mantém-se dentro de pequena faixa de variação, já os gêneros e, principalmente, as espécies, variam dentro de uma faixa mais ampla. As famílias com maiores riquezas nos cerrados analisados estão, de acordo com Joly (1977), Lawrence (1973) e Heywood (1978), entre as que possuem os maiores números de espécies na flora angiospérmica mundial, exceções feitas às Malpighiaceae e às Bignoniaceae.

Dos grupos de dez e onze gêneros mais importantes em cada área do cerrado, Chamaechrista, Hyptis, Paspalum e Vernonia estáo entre os mais representados e contêm mais de duzentas espécies em todo o mundo. Embora bem representados nas floras estudadas, nem todos os gêneros têm expressão significativa na vegetação do cerrado.

A flora estudada neste trabalho é relativamente rica em espécies, gêneros e famílias, quando comparada com aquela obtida em outras áreas do cerrado, o que deve ser relevado em função da pequena extensão da Reserva e da sua localização numa mancha disjunta da área marginal do cerrado. Essa flora pode ser acrescida, principalmente de espécies herbáceas, visto existirem várias delas de ocorrência rara e de pequeno porte, o que dificulta a amostragem.

Componente arbustivo-arbóreo - A maioria das famílias do componente arbustivo-arbóreo da flora amostrada, ou 55,32\% delas (vinte e seis famílias), possui uma espécie (Figura 5). Essa porcentagem é superior àquela observada para a flora como um todo. 
Tabela 1 - Lista de espécies amostradas no levantamento florístico do cerrado na

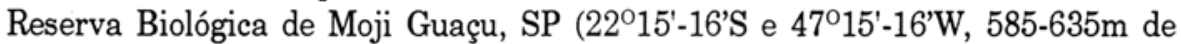
altitude). Forma de vida: Cam = caméfita, CAM-FAN = caméfita e fanerófita, EPI = epífita, $\mathrm{FAN}=$ fanerófita, $\mathrm{GEO}=$ geófita, $\mathrm{HEM}=$ hemicriptófita, LIA = liana, SPV = semi-parasita vascular e TER = terófita. Período de floração: $01=$ janeiro, 02 = fevereiro, $\ldots, 11$ = novembro e 12 = dezembro. Síndorme de dispersão: $\mathrm{ANE}=$ anemocórica, AUT = autocórica e ZOO = zoocórica.

\begin{tabular}{lccc}
\hline & FORMA & PERÍODO & SINDROME \\
FAMÍLIA/ESPÉCIE & DE & DE & DE \\
& VIDA & FLORAÇĀO & DISPERÇÃO \\
\hline
\end{tabular}

\section{PTERIDOPHYTA}

Polypodiaceae

Adianthum fovearum Raddi

Doryopteris concolor (Langsd. \& Fich.) Kuhn

Polypodium latipes Langsd. \& Fich.

Schizaeaceae

Anemia flexuosa (Sav.) Sw.

\section{ANGIOSPERMAE}

Monocotyledoneae

Bromeliaceae

Acanthostachys strobilacea (Sch.) Klotz.

Aechmea bromeliifolia (Rudge) Baker

Ananas ananassoides (Baker) L.B. Smith

Dyckia linearifolia $\mathrm{Mez}$

Commelinaceae

Commelina elegans H.B.K.

C. erecta L.

Dichorisandra hexandra Standl.

Cyperaceae

Bulbostylis capillaris (L.) C.B. Clarke

B. junciformis Kuntze

B. hirtella (Schrad.) Urban

B. juncoides (Vahl) Künkenth

B. sphaerocephala (Boeck.) C.B. Clarke

Cyperus cayennensis (Lam.) Britton

C. diffusus Vahl

C. ferax L.C. Rich

C. sesquiflorus (Torrey) Matf.

Rhynchospora exaltata Kunth.

R. glauca Vahl

R. nervosa (Vahl) Boeck

Scleria bracteata Cav.

S. comosa (Nees) Steud

$\begin{array}{lll}\text { HEM } & 08-11 & \text { ANE } \\ \text { HEM } & 02 & \text { ANE } \\ \text { HEM } & 11-05 & \text { ANE } \\ \text { HEM } & 01-04 & \text { ANE }\end{array}$

$\begin{array}{lll}\text { EPI } & 11-01 & \text { AUT } \\ \text { EPI } & 10 & \text { AUT } \\ \text { HEM } & 10 & \text { ZOO } \\ \text { EPI } & 09 & \text { AUT } \\ \text { TER } & 11-04 & \text { AUT } \\ \text { TER } & 11-03 & \text { AUT } \\ \text { HEM } & 01 & \text { AUT } \\ \text { HEM } & 11-01 & \text { AUT } \\ \text { HEM } & 03 & \text { AUT } \\ \text { HEM } & 03 & \text { AUT } \\ \text { HEM } & 11-05 & \text { AUT } \\ \text { HEM } & 11-04 & \text { AUT } \\ \text { HEM } & 10-05 & \text { AUT } \\ \text { HEM } & 11-06 & \text { AUT } \\ \text { HEM } & 12 & \text { AUT } \\ \text { HEM } & 01 & \text { AUT } \\ \text { HEM } & 04-08 & \text { AUT } \\ \text { HEM } & 12 & \text { AUT } \\ \text { HEM } & 01 & \text { AUT } \\ \text { HEM } & 08-10 & \text { AUT } \\ \text { HEM } & 10-05 & \text { AUT }\end{array}$


CONTINUAÇĀO

FAMÍLIA/ESPÉCIE

$\begin{array}{ccc}\text { FORMA } & \text { PERÍODO } & \text { SINDROME } \\ \text { DE } & \text { DE } & \text { DE } \\ \text { VIDA } & \text { FLORAÇĀO } & \text { DISPERÇĀO }\end{array}$

Dioscoriaceae

Dioscorea amaranthoides Presl.

LIA $\quad 01$

ANE

Gramineae

Andropogon angustatus (Presl.) Steud.

HEM

03-05

AUT

A. leucostachys H.B.K

HEM

$11-01$

HEM

12.01

Aristida riparia Cav.

HEM

$12-05$

Axonopus aureus Beauv.

HEM

04

HEM $\quad 12.05$

A. barbigerus (Kunth) Hitch.

HEM

11.05

A. suffultus (Mikan) Parodi

HEM

$02-05$

HEM

$01-04$

Digitaria neesiana Henr.

HEM

11

Echinolaena inflexa (Poir.) Chase

Eragrostis articulata (Schrank) Nees

HEM

E. pilosa (L.) Beauv.

TER

11-04

AUT

AUT

E. solida Nees

TER

04

ANE

AUT

AUT

AUT

AUT

Gymnopogon spicatus (Spreng.) Kuntz

Hyparrhenia rufa (Nees) Stapf

HEM

01

HEM

02-04

$01-04$

HEM

04

Ichnanthus procurrens (Nees) Swallen

I. sericeus Hack

HEM

09.04

ZOO

Leptocoryphium lanatum (H.B.K.) Nees

HEM

$01-06$

HEM

$09-01$

Melinis minutiflora Beauv.

HEM

11

HEM

01-06

HEM

01.02

P. cervicatum Chase

P. olyroides H.B.K.

HEM

P. parvifolium Lam.

Paspalum carinatum H.B.K.

HEM

HEM

10.04

11

AUT

$P$. gardnerianum Nees

HEM

09

AUT

AUT

$P$. guttatum Trin.

HEM

$11-04$

AUT

AUT

ANE

$P$. pectinatum Nees

HEM

04

01

AUT

P. plicatulum Michx.

HEM

10-04

P. polyphyllum Nees

P. sanguinolentum Trin.

HEM

HEM

Rhynchelitrum repens (Nees) C.E. Hubb.

TER

Schyzachirium condensatum (Kunth) Nees

HEM

S. lactiflorum (Hack.) Herter

HEM

04

AUT

AUT

ANE

ANE

AUT

AUT

AUT

AUT

AUT

AUT

S. sanguineum (Retz) Alst.

HEM

AUT

AUT

AUT

S. spicatum (Spreng.) Herter

Setaria gracilis Kunth

Sorghastrum minarum (Nees) Kunth

HEM

TER

10

AUT

11-04

AUT

04

ANE

ANE

03

04.05

ANE

01-04

ANE

HEM

$10-05$

ANE

HEM

$12-04$

AUT

Trachypogon macroglossus Trin.

HEM 
CONTINUAÇĀO

FAMÍLIA/ESPÉCIE

$\begin{array}{ccc}\text { FORMA } & \text { PERÍODO } & \text { SINDROME } \\ \text { DE } & \text { DE } & \text { DE } \\ \text { VIDA } & \text { FLORAÇĀO } & \text { DISPERÇÄO }\end{array}$

Iridaceae

Cipura paludosa Aubl.

GEO

01.03

AUT

Sisyrinchium incurvatum Gardn.

GEO

09

GEO

09-06

AUT

$S$. vaginatum Spreng.

GEO

09

AUT

Trimezia juncifolia (Kl.) Kunth

LIA

$08-10$

AUT

Smilax spinosa Mill.

Z00

Orchidaceae

Cleistis uliginosa Pabst.

GEO

01

HEM

01-03

ANE

Epidendrum denticulatum Rodr.

GEO

01

ANE

Galeandra junceoides Rodr.

GEO

03

ANE

$G$. montana Rodr.

GEO

03

ANE

Habenaria aff. gustavi-edwallii Hoehne

EPI

08

ANE

Ionopsis utricularioides (Sw.) Lind.

GEO

ANE

Pelexia laminata Schltr.

ANE

Palmae

Acanthococos emensis Toledo

GEO

04

$\mathrm{ZOO}$

Acrocomia aculeata (Jacquin) Loddiges ex Martius

FAN

10

$\mathrm{ZOO}$

Attalea geraxesis Barb. Rodr.

GEO

$-$

$\mathrm{ZOO}$

Butia paraguayensis (Barb. Rodr.) Bailey

FAN

11

$\mathrm{ZOO}$

Syagrus flexuosa (Mart.) Becc.

FAN

$05-06$

11.06

$\mathrm{ZOO}$

Dicotyledoneae

Acanthaceae

Ruellia geminiflora H.B.K

HEM

$09-02$

AUT

Amaranthaceae

Alternanthera brasiliana (L.) Kuntze

HEM

03

AUT

Froelichia lanata Moq.

HEM

11.02

AUT

Gomphrena macrorhiza Mart.

HEM

$09-04$

AUT

G. officinalis Mart.

HEM

$10-06$

AUT

G. virgata Mart.

HEM

08-09

AUT

Pfaffia gnaphaloides Mart.

HEM

10-11

AUT

$P$. jubata Mart.

HEM

09

AUT

Anacardiaceae

Anacardium humile St. Hil

Schinus therebinthifolius Radd.

CAM-FAN $\quad 08-10 \quad$ ZOO

FAN

$\mathrm{ZOO}$

Tapirira guianensi Aubl.

FAN

$09-10$

Z00

Annonaceae

Annona coriacea Mart.

CAM

11-04

$\mathrm{ZOO}$

A. cornifolia St. Hil.

FAN

11

$\mathrm{ZOO}$

A. crassiflora Mart.

FAN

10

$\mathrm{ZOO}$

Duguetia furfuracea (St. Hil.) Benth. \& Hook.

FAN

08-04

AUT 
CONTINUAÇĀO

FAMÍLIA/ESPÉCIE

$\begin{array}{ccc}\text { FORMA } & \text { PERÍODO } & \text { SINDROME } \\ \text { DE } & \text { DE } & \text { DE } \\ \text { VIDA } & \text { FLORAÇÃO } & \text { DISPERÇÃO }\end{array}$

Xylopia aromatica St. Hil

Apocynaceae

Aspidosperma tomentosum Mart.

FAN

FLORAÇĀO DISPERÇÃO

Hancornia speciosa Gomez

Himatanthus obovata (M. Arg.) Woods.

Macrosiphonia longiflora M.Arg.

FAN $03 \quad$ ZOO

M. petraea (St. Hil.) K. Sch.

M. velame M. Arg.

FAN

10

ANE

FAN

$\mathrm{ZOO}$

FAN

$-$

ANE

HEM

02-04

ANE

HEM

01

ANE

M. virescens M. Arg.

HEM

$-$

ANE

Mandevilla erecta (Vell.) Woods.

M. illustris (Vell.) Woods.

HEM

01.03

ANE

HEM

11-12

$12-01$

ANE

HEM

11

ANE

M. velutina (Mart.) Woods.

09.02

Mesechites mansoana (A. DC.) Woods.

HEM

$12-03$

ANE

LIA

$02-04$

Odontadenia lutea (Vell.) Markgr.

Prestonia riediellii (M.Arg.) Markgr.

Rhodocalyx rotundifolius M. Arg.

Temnadenia violacea (Vell.) Miers

LIA

LIA

HEM

01

ANE

ANE

ANE

LIA

$10-01$

ANE

$12-02$

ANE

\section{Araliaceae}

Didymopanax macrocarpum (C.\& S.) Seem

FAN

01.03

$01-05$

Z00

D. vinosum (C.\& S.) March.

FAN

$\mathrm{ZOO}$

Aristolochiaceae

Aristolochia esperanzae Kuntze

A. labiata Willd.

LIA

$01-05$

ANE

LIA

08-10

ANE

Asclepiadaceae

Astephanus gardneri Four.

Blepharodon lineare (Dcne.) Dcne.

LIA

04

HEM $\quad 11.04$

B. nitidum (Vell.) Macbr.

LIA

10.05

Chtamalia purpurea Dene.

HEM

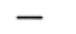

HEM $\quad 10.06$

Hemipogon setaceus Done.

HEM

12-01

LIA

01.05

ANE

ANE

ANE

ANE

Nautonia nummularia Dene.

ANE

Oxypetalum appendiculatum Mart. \& Zucc.

HEM

09-11

HEM

09-11

A. arvense (Vell.) Stellf.

CAM

12.03

Bignonia exoleta Vell.

LIA

09

LIA

04

FAN

11

Cybistax antisyphillitica Mart.

LIA

11.04

CAM

08-12

ANE

ANE

Jacaranda caroba A.EDC

ANE

ANE

ANE

ANE

ANE

ANE

ANE

ANE 
CONTINUAÇĀO

\begin{tabular}{lccc}
\hline & FORMA & PERÍODO & SINDROME \\
FAMÍLIA/ESPÉCIE & DE & DE & DE \\
& VIDA & FLORAÇĀO & DISPERÇĀO \\
\hline
\end{tabular}

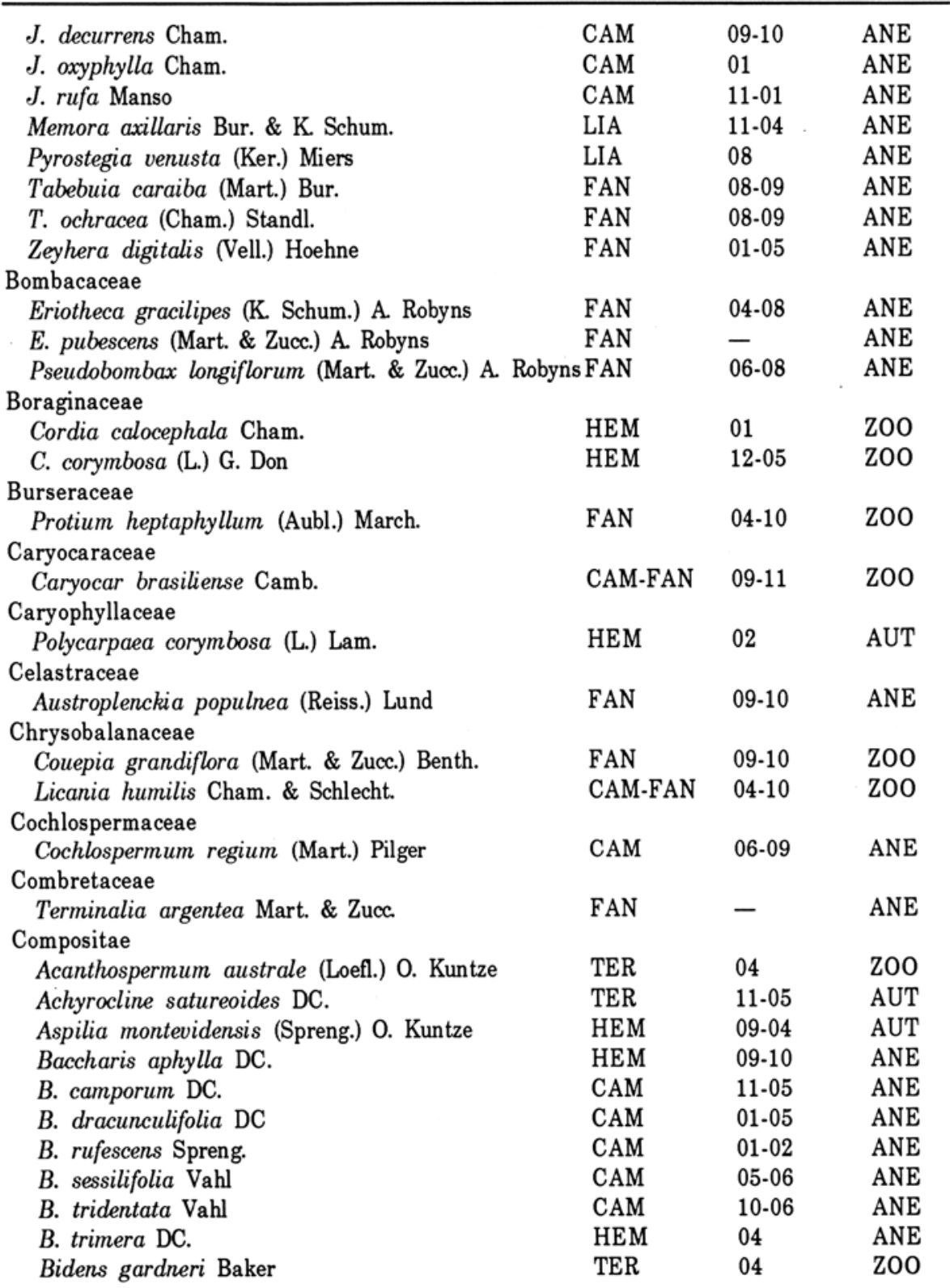


CONTINUAÇÄO

\section{FAMÍLIA/ESPÉCIE}

$\begin{array}{ccc}\text { FORMA } & \text { PERÍODO } & \text { SINDROME } \\ \text { DE } & \text { DE } & \text { DE } \\ \text { VIDA } & \text { FLORAÇĀO } & \text { DISPERÇĀO }\end{array}$

B. graveolens Mart.

Calea cuneifolia DC.

C. platylepis Sch. Bip.

Chaptalia integerrima (Vell.) Burk.

Dimerostemma lippioides (Baker) Baker.

Elephantopus micropappus Less.

E. mollis L.

E. racemosus Gardn.

Elephanthosis biflora Less.

Emilia sonchifolia DC.

Erechtites hieracifolia (L.) Raffin

Erigeron bonariensis L.

Eupatorium amigdalinum Kam.

E. campestre DC.

E. chlorolepsis Baker

E. dictyophyllum DC.

E. horminoides (DC.) Baker

E. intermedium DC.

E. laevigatum Lam.

E. lanigerum Hook.

E. maximiliani Schrad.

E. megacephalum Mart.

E. molissimum Baker

E. oxylepis DC.

E. purpurascens Sch. Bip

E. squalidum DC.

E. stachyophyllum Spreng.

Eremanthus sphaerocephalus Baker

Gochnatia barrosii

G. pulchra Cabrera

Inulopsis scaposa (Baker) Hoffm.

Mikania cordifolia (L.) Willd.

M. officinalis Mart.

M. sessilifolia DC.

Orthopappus angustifolius (Sw.) Gleason

Piptocarpha rotundifolia (Less.) Baker

Pseudogynoxys pohlii (Sch. Bip.) Leitāo F

Pterocaulon alopecurioides (Lam.) DC.

P. rugosum (Vahl) Malme

Riencourtia oblongifolia Gardn.

Senecio trixoides Gardn.
HEM

HEM

HEM

HEM

HEM

HEM

HEM

HEM

HEM

HEM

TER

TER

TER

HEM

HEM

HEM

HEM

CAM

HEM

CAM

HEM

CAM

HEM

HEM

CAM

HEM

TER

HEM

HEM

CAM

CAM

HEM

LIA

LIA

HEM

HEM

FAN

TER

HEM

HEM

HEM

TER

01.02

04

10-11

11.02

09-02

03

04-05

02-04

03

03-04

10-04

09

10-05

08-11

10-04

02-04

11

04-05

03

03-04

03

04

01-04

12-01

04

08

03-08

09.02

06. 08

05-08

04-05

10

02-04

10-02

04-05

01-09

12-01

04-07

01-02

03-05

12-04

08
$\mathrm{ZOO}$

Z00

AUT

AUT

AUT

ANE

ANE

ANE

ANE

ANE

ANE

ANE

ANE

ANE

ANE

ANE

ANE

ANE

ANE

ANE

ANE

ANE

ANE

ANE

ANE

ANE

ANE

ANE

ANE

ANE

ANE

ANE

ANE

ANE

ANE

ANE

ANE

ANE

ANE

ANE

AUT

ANE 
CONTINUAÇĀO

FAMÍLIA/ESPÉCIE

$\begin{array}{ccc}\text { FORMA } & \text { PERÍODO } & \text { SINDROME } \\ \text { DE } & \text { DE } & \text { DE } \\ \text { VIDA } & \text { FLORAÇĀO } & \text { DISPERÇÃO }\end{array}$

Stevia cinerascens Sch. Bip.

S. comixta Rob.

HEM

HEM

04

S. veronicae DC.

HEM

11.01

ANE

Trichogonia salviaefolia Gardn.

TER

03-04

ANE

Trixis glutinosa D.Don

01.04

ANE

T. verbasciformis Less.

06-08

ANE

Verbesina sordescens DC.

HEM

05

ANE

Vernonia apiculata Mart.

HEM

HEM

12.02

ANE

$V$. bardanoides Less.

HEM

03.04

AUT

HEM

01.04

V. brevifolia Less.

HEM

$09-05$

ANE

V. brevipetiolata Sch. Bip.

HEM

$12-03$

ANE

$V$. cognata Less

$10-03$

$V$. coriacea Less.

HEM

04

ANE

$V$. ferruginea Less.

HEM

V. glabrata Less.

CAM

08

ANE

V. grandiflora Less.

HEM

01

HEM

$10-01$

ANE

$V$. herbacea (Vell.) Rusby

$09-04$

HEM

V. holosericea Mart.

$05-08$

V. ignobilis Less

CAM

V. laevigata Mart.

HEM

10-04

CAM

V. lappoides Baker

HEM

04

V. megapotamica Spreng.

HEM

01-04

ANE

ANE

ANE

ANE

$V$. mucronulata Less.

CAM

11

ANE

$V$. onopordioides Baker

HEM

V. rubriramea Mart.

CAM

$V$. tragiaefolia DC.

HEM

HEM

\section{8}

ANE

ANE

ANE

V. virgulata Mart.

V. zuccariniana Mart.

CAM

Viguiera discolor Baker

HEM

\section{4}

ANE

ANE

ANE

ANE

ANE

10

ANE

ANE

$$
04
$$

ANE

HEM

09-02

AUT

V. robusta Gardn.

$$
\text { 03-05 }
$$

AUT

HEM

01-03

AUT

W. paludosa DC.

HEM

11

AUT

W. subvelutina DC.

HEM

03

AUT

Wulffia stenoglossa DC.

HEM

01

AUT

Connaraceae

Connarus suberosus Planch.

FAN

\section{4}

Z00

Convolvulaceae

Evolvulus sericeus $\mathrm{Sw}$.

HEM

12-01

AUT

Ipomoea virgata Meissn.

HEM

11.04

ANE

Jacquemontia sphaerocephala Meissn.

HEM

$12-03$

Merremia contorquens (Choisy) Hall.

HEM

01-12

AUT

HEM

11

AUT

LIA

M. macrocalyx (R. \& P.) O'Don 
CONTINUAÇĀO

FAMÍLIA/ESPÉCIE

$\begin{array}{ccc}\text { FORMA } & \text { PERÍODO } & \text { SINDROME } \\ \text { DE } & \text { DE } & \text { DE } \\ \text { VIDA } & \text { FLORAÇĀO } & \text { DISPERÇĀO }\end{array}$

Cucurbitaceae

Cayaponia espelina (Cogn.) Manso

LIA

11.04

$\mathrm{ZOO}$

Ceratosanthes hilariana Cogn.

LIA

09

$\mathrm{ZOO}$

Melancium campestre Naud.

HEM

02

$\mathrm{ZOO}$

Dilleniaceae

Davilla eliptica St. Hil.

FAN $\quad 03-04 \quad$ AUT

Ebenaceae

Diospyros hispida DC..

Erythroxylaceae

Erythroxylum ambiguum Peyr.

E. campestre St. Hil.

E. cuneifolium (Mart.) O.E.Schultz

E. deciduum St. Hil.

E. suberosum St. Hil.

E. tortuosum Mart.

$\begin{array}{lll}\text { FAN } & 10 & \text { ZOO }\end{array}$

FAN

$\mathrm{ZOO}$

FAN $\quad 09.06 \quad$ ZOO

FAN $\quad 08-12 \quad$ ZOO

FAN - $\quad$ ZOO

FAN $\quad 09-11 \quad$ ZOO

FAN $\quad 06-11 \quad$ ZOO

Euphorbiaceae

Bernardia spartioides (Baill.) M.Arg.

Croton eriocladus M.Arg.

C. glandulosus M. Arg.

C. grandivelum M. Arg.

C. lundianus M. Arg.

C. pohlianus M. Arg.

C. sclerocalyx M. Arg.

Euphorbia caecorum Mart.

Julocroton humilis Diedr.

J. lanceolatus M.Arg.

Manihot tripartita M. Arg.

Pera obovata Baill.

Phyllanthus orbiculatus M. Arg.

Sapium glandulatum (Vell.) Pax

Sebastiania bidentata (Mart.) Pax

S. serrulata M. Arg.

Tragia bahiensis M. Arg.

T. uberabana M. Arg.

Flacourtiaceae

Casearia sylvestris Sw.

Gentianaceae

Calolisianthus speciosus Gilg.

Dejanira foliosa (Griseb.) Guimarães

HEM

09-11

AUT

TER

$01-12$

AUT

TER

12

AUT

HEM

10-04

AUT

TER

04

AUT

HEM 09-05

AUT

HEM $\quad 11.05$

AUT

HEM 06-11 AUT

HEM 09-11 AUT

HEM 10-02 AUT

HEM 09.02 AUT

FAN 08 AUT

TER

11-04

AUT

HEM 11 AUT

HEM 10.04 AUT

TER 01-12 AUT

HEM 03 AUT

HEM 12

AUT

$\begin{array}{lll}\text { FAN } & 05-09 \quad \text { ZOO }\end{array}$

HEM 04

AUT

HEM 04 AUT

Gesneriaceae

Sinningia allagophylla (Mart.) Wiehler

HEM

10-02

ANE

S. splendens (Van Houte) O. Kuntze

HEM

01

ANE 
CONTINUAÇÃO

FAMÍLIA/ESPÉCIE

$\begin{array}{ccc}\text { FORMA } & \text { PERÍODO } & \text { SINDROME } \\ \text { DE } & \text { DE } & \text { DE } \\ \text { VIDA } & \text { FLORAÇĀO } & \text { DISPERÇÃO }\end{array}$

Guttiferae

Kielmeyera coriacea (Spr.) Mart.

FAN

$09-12$

ANE

K. rubriflora Camb.

FAN

03

ANE

K. variabilis Mart.

FAN

09

ANE

Hippocrateaceae

Peritassa campestris (Camb.) A. C. Smith

FAN

08-09

ANE

Labiatae

Eriope crassipes Bent.

HEM

08-03

AUT

Hyptis caespitosa St. Hil.

HEM

$11-04$

AUT

H. crinita Benth.

HEM

06-09

AUT

H. eriophylla Pohl.

HEM

01-03

AUT

H. marruboides Epl.

HEM

02-05

AUT

H. multibracteata Benth.

HEM

08

AUT

$H$. nudicaulis Benth.

HEM

01-04

AUT

H. pauliana Epl.

10-06

AUT

H. virgata Bent.

HEM

04-10

AUT

Peltodon tomentosus Pohl.

HEM

11-04

AUT

Salvia rosmarinoides St. Hil.

10-04

AUT

Lacistemataceae

Lacistema hasslerianum Chodat

FAN $\quad 04$

AUT

Lauraceae

Ocotea pulchella Mart.

FAN

11-01

$\mathrm{ZOO}$

Leguminosae - Caesalpinioideae

Bauhinia rufa Steud.

FAN

10-01

AUT

Chamaechrista basifolia (G. Don) I. \& B.

CAM

01-03

AUT

C. calycioides (Collad.) Greene

HEM

01.02

AUT

C. cathartica (Mart.) I. \& B.

08-05

AUT

C. desvauxii (Collad.) Killip

CAM

02-04

C. fasciculata (Michx.) Greene

01

AUT

C. flexuosa (L.) Greene

HEM

11-04

AUT

CAM

AUT

C. labouriaea (I. \& B.) I. \& B.

11-02

AUT

C. nictitans (L.) Moench.

CAM

01-04

AUT

C. trichopoda (Benth.) Britton \& Rose

12-01

AUT

Copaifera langsdorffii Desf.

HEM

02

$\mathrm{ZOO}$

Dimorphandra mollis Benth.

Hymenaea stigonocarpa Mart.

FAN

11-12

$\mathrm{ZOO}$

FAN

09-10

$\mathrm{ZOO}$

FAN

$12 \cdot 02$

ANE

Senna pilifera (Vog.) I. \& B.

CAM

11

AUT

S. rugosa (G. Don) I. \& B.

FAN

01-04

AUT

S. silvestris (Vell.) I. \& B.

FAN

01

AUT 
FAMÍLIA/ESPÉCIE

\begin{tabular}{ccc} 
FORMA & PERÍODO & SINDROME \\
DE & DE & DE \\
VIDA & FLORAÇĀO & DISPERÇĀO \\
\hline
\end{tabular}

Faboideae

Acosmium dasycarpum (Vog.) Yakol.

A. subelegans (Mohl.) Yakol.

FAN

$11-01$

ANE

Aeschynomene falcata Benth.

FAN

$11-01$

ANE

A. hystrix Poir.

A. marginata Benth.

Andira laurifolia Benth.

Bowdichia virgilioides H.B.K.

Calopogonium caeruleum Hemsl.

Camptosema ellipticum (Desv.) Bork

HEM

10-02

TER

$01-04$

$\mathrm{ZOO}$

TER

10.01

$\mathrm{ZOO}$

CAM

09-10

$\mathrm{ZOO}$

FAN

06.09

$\mathrm{ZOO}$

HEM

02

ANE

LIA

08.09

AUT

Centrosema angustifolium H.B.K.

C. bracteosum Benth.

LIA

$01-04$

AUT

C. venosum Mart.

HEM

10.02

AUT

Clitoria guyanensis Benth.

LIA

$02-03$

AUT

Crotalaria maypurensis H.B.K.

C. foliosa Benth.

HEM

10-12

AUT

TER

10.06

AUT

C. pohliana Benth.

TER

Dalbergia miscolobium Benth.

Desmodium guaraniticum (Schindl.) Malme

HEM

01.05

AUT

FAN

D. incanum (Sw.) DC.

HEM

10.04

AUT

D. pachyrrizum Vog.

TER

10

AUT

Eriosema campestre Benth.

HEM

HEM

HEM

E. crinitum (H.B.K.) G. Don

HEM

E. heterophyllum Benth.

HEM

E. longifolium Bent

Galactia decumbens (Benth.) Hassler

HEM

01

ANE

G. Martii DC.

HEM

Harpalyce brasiliana Benth.

FAN

$01-04$

$\mathrm{ZOO}$

Indigofera gracilis Bong.

HEM

Machaerium acutifolium Vog.

M. villosum Vog.

Macroptilium prostratum (Benth.) Urban

FAN

FAN

$$
01
$$

$\mathrm{ZOO}$

Platypodium elegans Vog.

LIA

Poiretia coriifolia Vog

FAN

CAM

$09-03$

$\mathrm{ZOO}$

09.05

AUT

$01-12$

AUT

10-03

AUT

12-04

$11-05$

AUT

AUT 12-04

04

AUT

08-12

AUT

11

AUT

ANE

11-12 ANE

Rhynchosia melanocarpa Grear

Stylosanthes gracilis H.B.K.

S. guianensi Sw.

LIA

TER

TER

S. scabra Vog.

Tephrosia rufescens Benth.

Vatairea macrocarpa (Benth.) Ducke

Vigna peduncularis (H.B.K.) Fawcett et Rendle

HEM

HEM

09-12

AUT

$-$

ANE

FAN

Zornia latifolia $\mathrm{Sm}$.

Z. reticulata $\mathrm{Sm}$.

HEM

01

AUT

$02-08$

ZOO

09-04 AUT

12.05 AUT

06-08 AUT

HEM

10-02

AUT

HEM

$-$

ANE

02-03 AUT

04

Z0O

10-02 ZOO 
CONTINUAÇĀO

\begin{tabular}{lccc}
\hline & FORMA & PERÍODO & SINDROME \\
FAMÍLIA/ESPÉCIE & DE & DE & DE \\
& VIDA & FLORAÇĀO & DISPERÇĀO \\
\hline
\end{tabular}

Mimosoideae

Anadenanthera falcata (Benth.) Speg.

Desmanthus depressus Humb. \& Bompl.

Enterolobium gummiferum (Mart.) Macbr.

Mimosa acerba Benth.

M. axillaris Benth.

M. calycina Benth.

M. capillipes Benth.

M. distans Benth.

M. eriophylla Bent.

$M$. invisa Mart

M. meticulosa Mart.

M. rixosa Mart.

M. subsericea Benth.

M. tremula Benth.

Stryphnodendron adstringens (Mart.) Coville

Loganiaceae

Strychnos pseudoquina St. Hil

Loranthaceae

Phrygilanthus eugenioides (H.B.K.) Eichl.

Psittacanthus robustus Mart.

Lythraceae

Cuphea thymoides Cham. \& Schlecht

Diplusodon virgatus Pohl

Lafoensia pacari St. Hil.

Malpighiaceae

Banisteriopsis anisandra (A. Juss.) Gates

B. argyrophylla (A. Juss.) Gates

B. campestris (A. Juss.) Little

B. laevifolia (A. Juss.) Gates

B. stellaris (Griseb.) Gates

B. variabilis Gates

Byrsonima coccolobifolia (Spr.) Kunth

B. intermedia A. Juss.

B. verbascifolia L.C. Rich

Camarea affinis St. Hil.

Heteropteris acutifolia A. Juss.

H. byrsonimaefolia A. Juss.

H. umbelata A. Juss.

Peixotoa reticulata Griseb

Tetrapteris longibracteata A. Juss.
FAN $\quad 09$

CAM 02

ANE

FAN -

CAM 01-04

CAM

09-11

03-06

CAM

CAM

11-04

CAM

CAM

CAM

CAM

CAM

CAM

CAM

FAN

10-01

01

01

01

01-02

12-04

10-02

09

01

FAN

SPV

SPV

12

02-06

CAM

10-04

01-04

04-05

FAN

LIA

LIA

LIA

LIA

LIA

LIA

FAN

FAN

FAN

HEM

FAN

FAN

LIA

LIA

CAM
05-08

04

10-04

11-05

01-04

11-05

11.06

10-04

08-11

12-02

10

09

11-04

05-11

09
$\mathrm{ZOO}$

AUT

AUT

ZOO

ANE

ZOO

ANE

ANE

ZOO

ANE

$\mathrm{ZOO}$

Z0O

ANE

ANE

$\mathrm{ZOO}$

Z00

$\mathrm{Z} 00$

AUT

AUT

AUT

ANE

ANE

ANE

ANE

ANE

ANE

$\mathrm{ZOO}$

$\mathrm{ZOO}$

$\mathrm{ZOO}$

ANE

ANE

ANE

ANE

ANE

ANE 
CONTINUAÇĀO

FAMÍLIA/ESPÉCIE

$\begin{array}{ccc}\text { FORMA } & \text { PERÍODO } & \text { SINDROME } \\ \text { DE } & \text { DE } & \text { DE } \\ \text { VIDA } & \text { FLORAÇĀO } & \text { DISPERÇĀO }\end{array}$

\begin{tabular}{|c|c|c|c|}
\hline \multicolumn{4}{|l|}{ Malvaceae } \\
\hline Krapovichasia macrodon (DC.) Fryx & HEM & $08-04$ & AUT \\
\hline Pavonia malacophylla Gurcke & CAM & 04 & AUT \\
\hline P. speciosa H.B.K. & HEM & 10.04 & AUT \\
\hline Sida aurantiaca St. Hil. & TER & 08.05 & AUT \\
\hline S. glaziovii K. Schum. & CAM & 04 & AUT \\
\hline S. linifolia Juss. & TER & $11-05$ & AUT \\
\hline S. rhombifolia L. & TER & 04 & AUT \\
\hline S. spinosa L. & TER & $10-02$ & AUT \\
\hline \multicolumn{4}{|l|}{ Melastomataceae } \\
\hline Acisanthera alsinaefolia Triana & CAM & 11 & ANE \\
\hline Cambessedesia ilicifolia (DC.) Triana & CAM & $08-11$ & ANE \\
\hline Leandra erostrata Cogn. & FAN & 12 & ANE \\
\hline L. lacunosa Cogn. & FAN & $05-10$ & ANE \\
\hline Miconia albicans Triana & CAM & 04 & $\mathrm{ZOO}$ \\
\hline M. langsforffii Cogn. & FAN & 11 & $\mathrm{ZOO}$ \\
\hline M. rubiginosa (Bonpl.) DC. & FAN & 01 & $\mathrm{ZOO}$ \\
\hline M. stenostachya DC. & CAM & $08-03$ & $\mathrm{ZOO}$ \\
\hline Tibouchina gracilis (Bonpl.) Cogn. & CAM & $11-02$ & ANE \\
\hline T. sebastianopolitana Cogn. & CAM & 03 & ANE \\
\hline T. stenocarpa (DC.) Cogn. & FAN & 03.08 & ANE \\
\hline \multicolumn{4}{|l|}{ Menispermaceae } \\
\hline Cissampelos ovalifolia R. \& P. & HEM & 09.02 & AUT \\
\hline \multicolumn{4}{|l|}{ Monimiaceae } \\
\hline Siparuna guianensis Aubl & FAN & 09 & $\mathrm{ZOO}$ \\
\hline \multicolumn{4}{|l|}{ Moraceae } \\
\hline Brosimum gaudichaudii Trec. & FAN & $09-10$ & $\mathrm{ZOO}$ \\
\hline Dorstenia tubicina R. \& P. & HEM & 02 & $\mathrm{ZOO}$ \\
\hline Ficus citrifolia P. Miller & FAN & 09 & $\mathrm{ZOO}$ \\
\hline \multicolumn{4}{|l|}{ Myristicaceae } \\
\hline Virola sebifera Aubl. & FAN & - & $\mathrm{ZOO}$ \\
\hline \multicolumn{4}{|l|}{ Myrsinaceae } \\
\hline Rapanea ferruginea (R. \& P.) Mez & FAN & 09 & $\mathrm{ZOO}$ \\
\hline R. guianensis Aubl & FAN & $05-12$ & $\mathrm{ZOO}$ \\
\hline \multicolumn{4}{|l|}{ Myrtaceae } \\
\hline Campomanesia pubescens (A.P.DC.) Berg. & FAN & 09 & $\mathrm{ZOO}$ \\
\hline Eugenia albo-tomentosa Camb. & FAN & $09-11$ & $\mathrm{ZOO}$ \\
\hline E. aurata Berg. & FAN & 10 & $\mathrm{ZOO}$ \\
\hline E. bimarginata DC. & FAN & 02 & $\mathrm{ZOO}$ \\
\hline E. livida Berg. & FAN & $05-08$ & $\mathrm{ZOO}$ \\
\hline E. observa Berg. & FAN & $08-10$ & $\mathrm{ZOO}$ \\
\hline E. pitanga (Berg.) Kiaersk. & FAN & 09 & $\mathrm{ZOO}$ \\
\hline
\end{tabular}


CONTINUAÇĀO

FAMÍLIA/ESPÉCIE

$\begin{array}{ccc}\text { FORMA } & \text { PERÍODO } & \text { SINDROME } \\ \text { DE } & \text { DE } & \text { DE } \\ \text { VIDA } & \text { FLORAÇĀO } & \text { DISPERÇĀO }\end{array}$

Hexachlamys humilis Berg.

Myrcia albo-tomentosa Camb.

M. bela Camb.

M. lingua Berg.

M. tomentosa (Aubl.) DC.

Psidium cinereum Mart.

$P$. grandifolium Mart.

$P$. incanescens Mart.

P. suffruticosum Berg.

Nyctaginaceae

Guapira noxia (Netto) Lund

Neea mollis Spr. ex Schum.

$N$. theifera Oersted

Ochnaceae

Ouratea spectabilis (Mart.) Engl.

Opiliaceae

Agonandra brasiliensis Miers.

Oxalidaceae

Oxalis physocallyx Zucc.

Passifloraceae

Passiflora lepidota Mast.

Polygalaceae

Monnina cuneata St. Hil.

Polygala angulata DC.

P. rhodoptera Mart.

P. timoutou Auvbl.

Securidaca tomentosa St. Hil.

Proteaceae

Roupala montana Aubl.

Rhamnaceae

Crumenaria polygaloides Reiss.

Rosaceae

Prunus sellowii $\mathrm{Sm}$.

Rubus brasiliensis Mart.

Rubiaceae

Alibertia macrophylla K. Schum.

A sessilis (Vell.) K. Schum.

Borreria eupatorioides Cham. \& Schlecht.

B. suaveolens Meyer

B. warmingii $\mathrm{K}$. Schum.

Coccocypselum lanceolatum (R. \& P.) Pers.
CAM

FAN

FAN

FAN

FAN

CAM

CAM

CAM

CAM

FAN

FAN

FAN

FAN

FAN

HEM

01-04

AUT

HEM

01-04

$\mathrm{Z} 00$

HEM

12

HEM

12.02

12.02

HEM

HEM

04

LIA

05-09

08.09

AUT

FAN

09-03

ANE

HEM

FAN

06-09

$\mathrm{ZOO}$

FAN

02

$\mathrm{ZOO}$

FAN

09-10

$\mathrm{ZOO}$

HEM/CAM $\quad 08-11$

$\mathrm{ZOO}$

HEM

03-08

AUT

HEM

09-06

AUT

HEM

04

AUT

HEM 
CONTINUAÇÃO

FAMÍLIA/ESPÉCIE

$\begin{array}{ccc}\text { FORMA } & \text { PERÍODO } & \text { SINDROME } \\ \text { DE } & \text { DE } & \text { DE } \\ \text { VIDA } & \text { FLORAÇĀO } & \text { DISPERÇĀO }\end{array}$

Coussarea hydrangeaefolia (Benth.) Benth. \& Hook. FAN

Declieuxia fruticosa (Willd.) 0. Kuntze

HEM

-

$\mathrm{ZOO}$

D. lysimachioides Zucc.

HEM

11.03

$\mathrm{ZOO}$

Diodia schumannii Standl.

TER

09.04

$\mathrm{ZOO}$

D. teres Walt.

TER

12-04

AUT

Guettarda viburnoides Cham. \& Schlecht.

FAN

10-11

AUT

Palicourea rigida H.B.K

CAM-FAN $\quad 10-05$

ZOO

Psychotria barbiflora DC.

05-12

$\mathrm{ZOO}$

P. carthaginensis Jacq.

CAM

$11-05$

$\mathrm{ZOO}$

P. tricholoba M. Arg.

CAM

$\mathrm{ZOO}$

Relbunium hirtum K. Schum.

CAM

10-04

$\mathrm{ZOO}$

$R$. noxium $\mathrm{K}$. Schum.

TER

01

$\mathrm{ZOO}$

Richardia brasiliensis Gomes

TER

02-04

$\mathrm{ZOO}$

Rudgea viburnoides (Cham.) Benth.

TER

12-04

$\mathrm{ZOO}$

Sabicea brasiliensis Wernh.

08-10

$\mathrm{ZO0}$

Tocoyena formosa (C. \& S.) K. Schum.

Rutaceae

Zanthoxylum fhoifolium Lam.

FAN

08-04

$\mathrm{ZOO}$

FAN

11-01

$\mathrm{ZOO}$

FAN $\quad-\quad$ ZOO

Sapindaceae

Magonia pubescens St. Hil.

FAN

06

ANE

Serjania erecta Radlk.

CAM

11-01

ANE

S. lethalis St. Hil.

LIA

08-09

ANE

S. ovalifolia Radlk.

01

ANE

S. reticulata Camb.

03-04

ANE

Sapotaceae

Chrysophyllum soboliferum Rizz.

10-11

$\mathrm{Z} 00$

Pouteria ramiflora (Mart.) Radlk.

HEM

09

$\mathrm{ZOO}$

Scrophulariaceae

Buchnera lavandulacea Cham. \& Schlecht.

HEM

04-08

ANE

B. lobelioides Cham.

HEM

10-12

ANE

Esterhazia splendida Mikan

HEM

04

ANE

Solanaceae

Cestrum pedicellatum Sendtn.

CAM

05-11

$\mathrm{ZOO}$

C. schlechtendalii G. Don

CAM

04

$\mathrm{ZOO}$

C. sendtenerianum Mart.

CAM

01-06

$\mathrm{ZOO}$

Solanum americanum Mill

TER

03

$\mathrm{ZOO}$

S. lycocarpum St. Hil.

CAM

09-11

$\mathrm{ZOO}$

S. palinacanthum Dunal

TER

10-03

$\mathrm{ZOO}$

S. paniculatum L.

CAM

11

$\mathrm{ZOO}$ 
CONTINUAÇÃO

FAMÍLIA/ESPÉCIE

$\begin{array}{ccc}\text { FORMA } & \text { PERÍODO } & \text { SINDROME } \\ \text { DE } & \text { DE } & \text { DE } \\ \text { VIDA } & \text { FLORAÇĀO } & \text { DISPERÇĀO }\end{array}$

Sterculiaceae

Byttneria sagittifolia St. Hil.

HEM

09-05

AUT

Helicteres brevispira St. Hil.

CAM

09

AUT

H. sacarolha St. Hil.

11.03

AUT

Melochia splendens St. Hil.

$11-12$

AUT

Waltheria communis $\mathrm{L}$.

09.05

AUT

W. indica $\mathrm{L}$.

11-04

AUT

Styracaceae

Styrax camporum Pohl

S. ferrugineus Nees \& Mart.

Symplocaceae

Symplocos pubescens Klotz.

Tiliaceae

Luehea divaricata Mart.

Turneraceae

Piriqueta aurea (Camb.) Urban

HEM

11

$\mathrm{ZOO}$

FAN

$02-06$

$\mathrm{ZOO}$

Umbelliferae

Eryngium junceum Cham. \& Schlecht.

FAN

02-04

Z00

E. pristis Cham. \& Schlecht.

FAN

08

AUT

FAN

09-11

AUT

HEM

\section{1-01}

AUT

Verbenaceae

Aegiphila lhotzkyana Cham.

Lantana camara L.

$\begin{array}{lll}\text { HEM } & 11-01 & \text { AUT } \\ \text { HEM } & 01 & \text { AUT }\end{array}$

L. fucata Lindl.

$\mathrm{ZOO}$

CAM

\section{4}

$\mathrm{ZOO}$

CAM

$10-01$

$\mathrm{ZOO}$

L. hypoleuca Briz.

03-05

$\mathrm{ZOO}$

Lippia lasiocalycina Cham.

CAM

$01-12$

AUT

L. lupulina Cham.

CAM

$01-12$

AUT

L. salviaefolia Cham.

03-04

AUT

L. stachyoides Cham.

CAM

11-02

AUT

Stachytarpheta gesnerioides Cham.

CAM

01-02

AUT

S. maximiliani Schauer

HEM

11-02

AUT

Vitaceae

Cissus inundata (Baker) Pl.

LIA

12-04

$\mathrm{ZOO}$

C. rosa Rich.

12-02

$\mathrm{ZOO}$

C. sessilifolia (Baker) Gilg.

LIA

11-01

$\mathrm{ZOO}$

Vochysiaceae

Qualea grandiflora Mart.

FAN

12

ANE

Q. multiflora Mart.

FAN

12

ANE

Q. parviflora Mart.

FAN

11

ANE

Vochysia cinamommea Pohl

FAN

04

ANE

V. tucanorum (Spr.) Mart.

FAN 


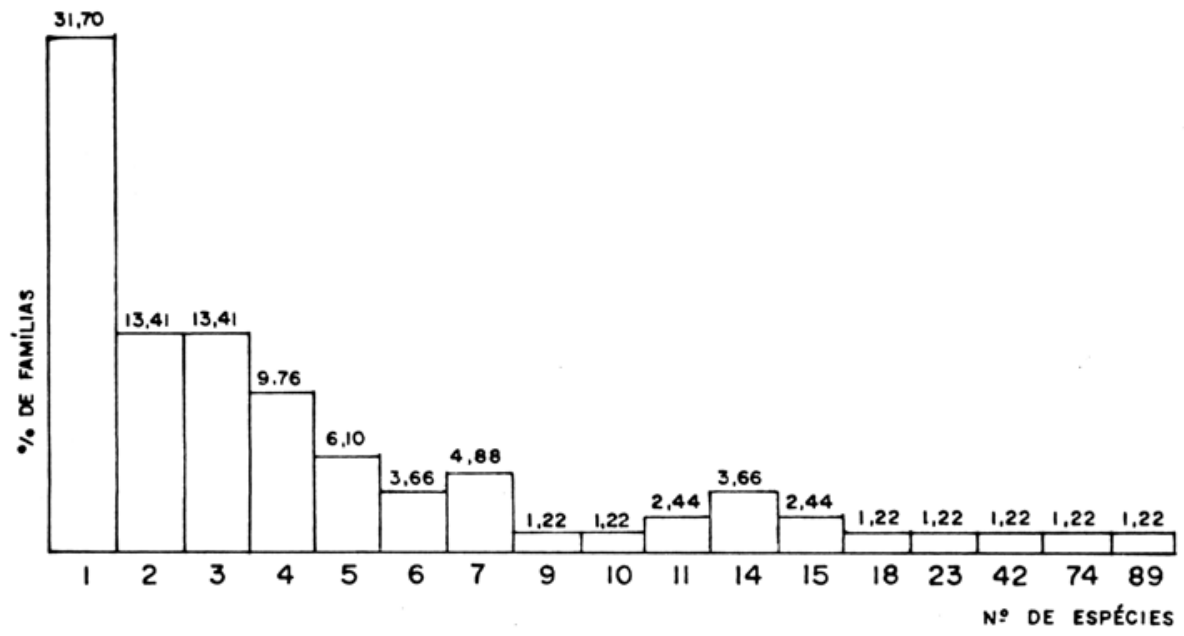

Figura 3 - Distribuição, em porcentagem, das famílias pelo número de espécies, no cerrado da Reserva Biológica de Moji Guaçu, SP $\left(22^{\circ} 15^{\prime}-16 \mathrm{~S}\right.$ e $47^{\circ} 08^{\prime}-12^{\prime} \mathrm{W}, 585-635 \mathrm{~m}$ de altitude).

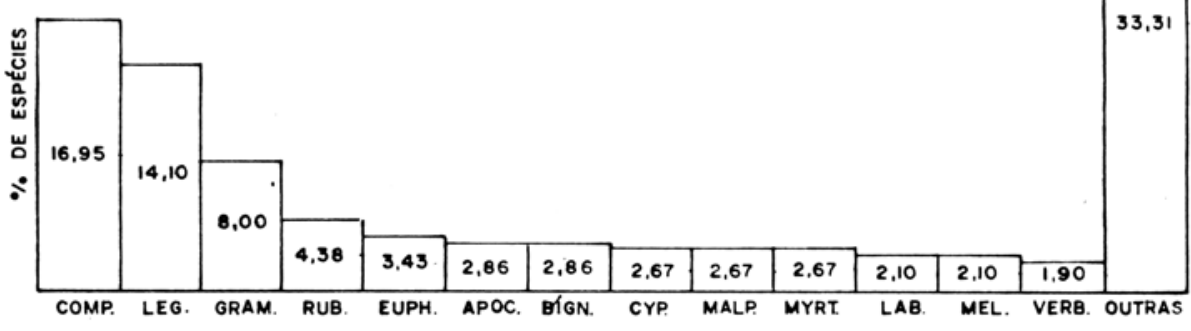

Figura 4 - Distribuição, em porcentagem, das espécies nas famílias mais representadas no cerrado da Reserva Biológica de Moji Guaçu, SP $\left(22^{\circ} 15^{\prime}-16\right.$ S e $47^{\circ} 08^{\prime}-12^{\prime} \mathrm{W}$, $585-635 \mathrm{~m}$ de altitude). Comp. = Compositae, Leg. = Leguminosae, Gram. = Gramineae, Rub. = Rubiaceae, Euph. $=$ Euphorbiaceae, Apoc. $=$ Apocynaceae, Bign. $=$ Bignoniaceae Cyp. $=$ Cyperaceae, Malp. = Malpighiaceae, Myrt. = Myrtaceae, Lab. = Labiatae, Mel. = Melastomataceae e Verb. = Verbenaceae. 


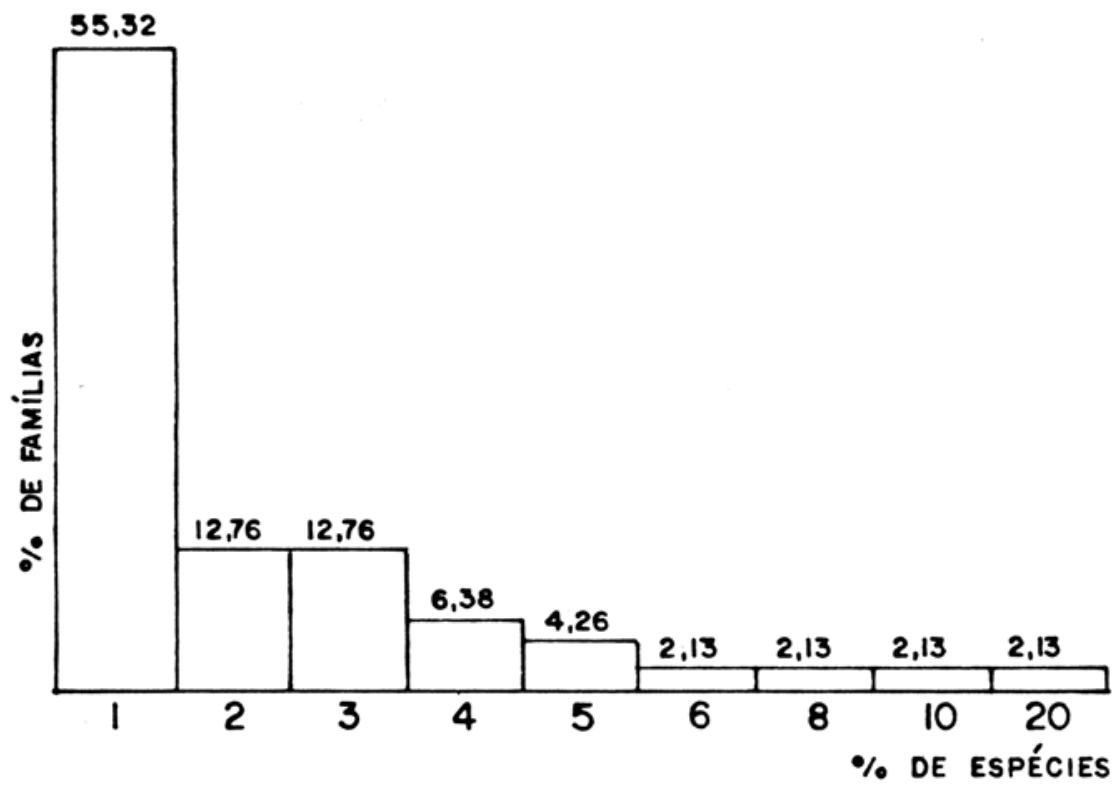

Figura 5 - Distribuição, em porcentagem, das famílias pelo número de espécies do componente arbustivo-arbóreo, no cerrado da Reserva Biológica de Moji Guaçu, SP $\left(22^{\circ} 15^{\prime}\right.$ $16 \mathrm{~S}$ e $47^{\circ} 08^{\prime}-12^{\prime} \mathrm{W}, 585-635 \mathrm{~m}$ de altitude).

As famílias com maiores riquezas são: Leguminosae, Myrtaceae, Rubiaceae, Erythroxylaceae, Malpighiaceae, Vochysiaceae, Melastomataceae, Bignoniaceae e Annonaceae, totalizando $54,11 \%$ das espécies desse componente (Figura 6).

Do total de famílias amostradas na flora como um todo, $35,36 \%$ (vinte e nove delas) possuem elementos exclusivos do componente arbustivo-arbóreo.

Comparando-se os dados obtidos com aqueles de outras áreas do cerrado (Goodland, 1969; Ratter, 1980 e Warming, 1892), observa-se uma variação de 45 famílias amostradas na Lagoa Santa e 50 amostradas no Triângulo Mineiro, 87 gêneros na Lagoa Santa a 128 no Triângulo Mineiro e de 122 espécies em Moji Guaçu a 187 na Lagoa Santa. Leguminosae, Myrtaceae, Malpighiaceae e Vochysiaceae são as famílias que ocorrem em todas as áreas, dentro do grupo mais representado.

Componente herbáceo-subarbustivo - As espécies herbáceo-subarbustivas do cerrado da Reserva compöem a maioria da sua flora, com 76,76\% do total (quatrocentas e duas). As famílias desse componente que possuem exclusivamente espécies caméfitas, hemicriptófitas, geófitas, terófitas e lianas representam $41,46 \%$ das famílias da flora como um todo. 


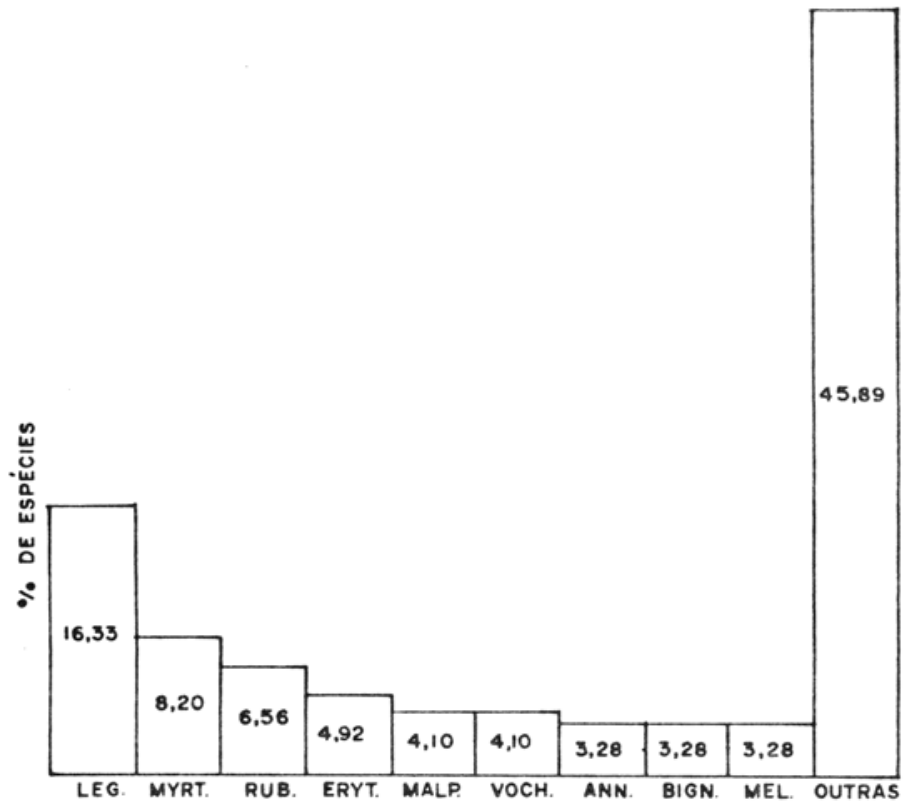

Figura 6 - Distribuição, em porcentagem,. das espécies do componente arbustivo-arbóreo nas famílias mais representadas no cerrado da Reserva Biológica de Moji Guaçu, SP $\left(22^{\circ} 15^{\prime}-16{ }^{\prime} \mathrm{S}\right.$ e $47^{\circ} 08^{\prime}-12^{\prime} \mathrm{W}, 585-635, \mathrm{~m}$ de altitude $)$. Leg. = Leguminosae, Myrt. = Myrtaceae, Rub. = Rubiaceae, Erty. = Erythroxylaceae, Malp. = Malpighiaceae, Voch. = Vochysiaceae, Ann. = Annonaceae, Bign. = Bignoniaceae, Mel. = Melastomataceae.

Das famílias amostradas, 32,69\% possuem uma única espécie (Figura 7), que é um valor próximo do obtido para a flora como um todo. Compositae, Leguminosae, Gramineae, Euphorbiaceae, Rubiaceae, Cyperaceae, Apocynaceae, Labiatae, Bignoniaceae, Verbenaceae, Malpighiaceae e Malvaceae são as famílias com os maiores números de espécies na área em estudo, somando $70,22 \%$ do total de espécies do componente campestre (Figura 8). Essas são as maiores famílias de angiospermas em todo o mundo e apenas Orchidaceae, dentre estas, é pouco representada na área.

Comparando-se os resultados desse trabalho com aqueles efetuados na Lagoa Santa (Warming, 1892) e em Brasília (Ratter, 1980), observa-se uma variação de 43 famílias em Brasília a 52 em Moji Guaçu e na Lagoa Santa, de 151 gêneros em Brasília a 220 na Lagoa Santa e de 290 espécies em Brasília à 640 na Lagoa Santa.

Os poucos trabalhos publicados sobre o componente herbáceo, indicam grandes mudanças na sua composição entre diferentes áreas do cerrado. Sua flora é mais rica que a do componente arbustivo-arbóreo. Quanto mais o cerrado se aproxima da sua fisionomia florestal (cerradão), menor será a riqueza da sua flora, que é composta por espécies heliófitas (Coutinho, 1978; Goodland, 1969). 


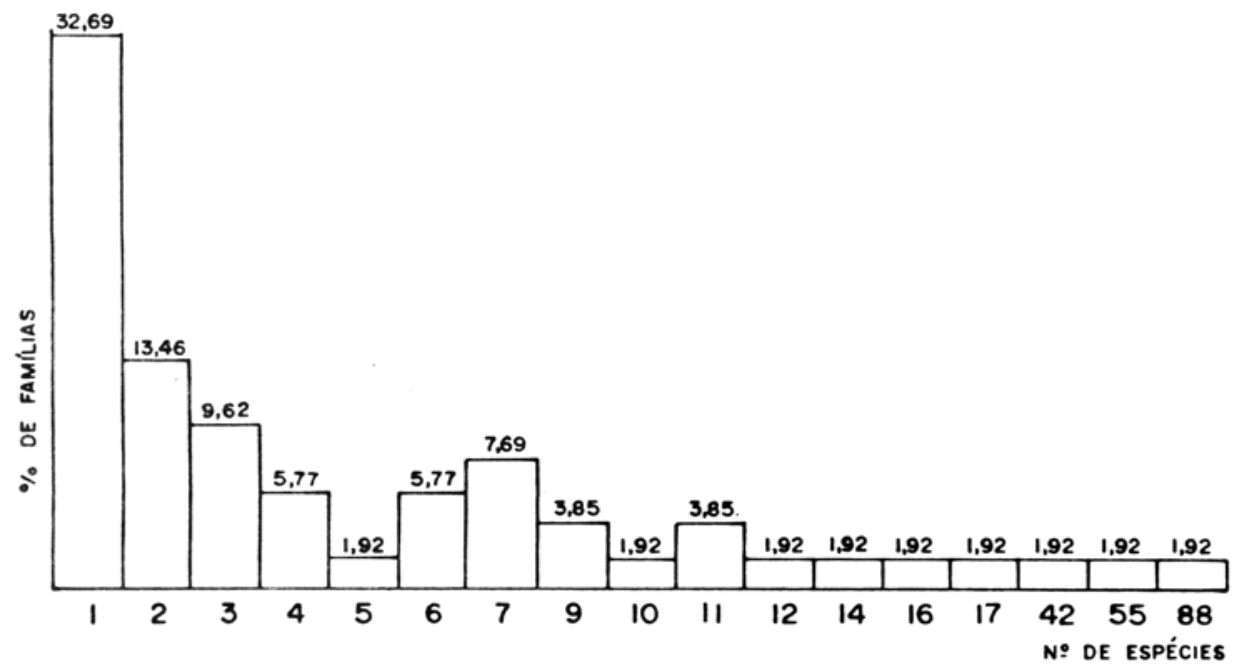

Figura 7 - Distribuição, em porcentagem, das famílias pelo número de espécies do componente herbáceo-subarbustivo, no cerrado da Reserva Biológica de Moji Guaçu, SP $\left(22^{\circ} 15^{\prime}-16 \mathrm{~S}^{\prime}\right.$ e $47^{\circ} 08^{\prime}-12^{\circ} \mathrm{W}, 585-635 \mathrm{~m}$ de altitude).

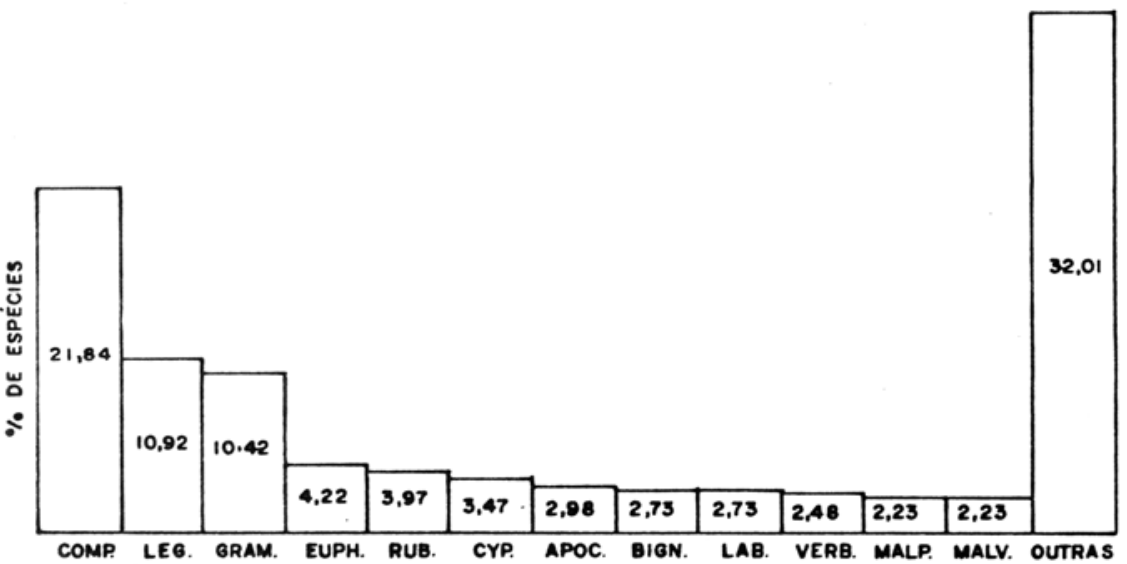

Figura 8 - Distribuição, em porcentagem, das espécies do componente herbáceosubarbustivo nas famílias mais representadas no cerrado da Reserva Biológica de Moji Guaçu, SP (22 $15^{\circ}-16$ 'S e $47^{\circ} 08^{\prime}-12^{\prime} \mathrm{W}, 585-635 \mathrm{~m}$ de altitude). Comp. = Compositae, Leg. = Leguminosae, Gram. $=$ Gramineae, Euph. $=$ Euphorbiaceae, Rub. $=$ Rubiaceae, Cyp. $=$ Cyperaceae, Apoc. $=$ Apocynaceae, Bign. $=$ Bignoniaceae, Lab. $=$ Labiatae, Verb. $=$ Verbenaceae, Malp. $=$ Malpighiaceae e Malv. $=$ Malvaceae. 
Outro fator importante para o pouco conhecimento da flora campestre do cerrado refere-se à sua sazonalidade anual, já que muitas de suas espécies têm comportamento epigeo similar ao de plantas de ciclo curto, com ramos de brotamento efêmeros. Uma discussão acerca das características desse componente do cerrado é apresentada por Mantovani (1990).

Comparando-se os números de espécies obtidas nos dois componentes do cerrado na Lagoa Santa, em Brasília e em Moji Guaçu, observa-se uma relação de 1:2 entre os componentes florestal e campestre, em Brasília e de 1:3 em Moji Guaçu e na Lagoa Santa. Nestas duas áreas há predomínio de fisionomias abertas do cerrado, ao passo que naquela ocorre, também, o cerradão.

Devido à ocorrência de fisionomias abertas na Reserva, a flora campestre teve maior influência nos resultados obtidos para o cerrado como um todo, como se observa no grupo de gêneros mais importantes obtidos na comparação entre diversas áreas.

A flora do componente arbustivo-arbóreo do cerrado apresenta composição menos variável que aquela do componente herbáceo-subarbustivo, que é mais sensível às mudanças de clima, solo, queimadas e diversos outros fatores que atuam nas regiōes de sua ocorrência, necessitando-se levantamentos florísticos intensos e sistemáticos em diversas áreas do cerrado e em diversas formações vegetais que com ele se relacionam, para que se possa conhecer adequadamente a sua flora e as ligações que mantém com outras formaçōes.

As informações acerca da síndrome de dispersão dos diásporos e do período de floração de cada espécie foram utilizadas para a análise das variações fenológicas (Mantovani \& Martins, 1988), o que indicou padrões diferentes entre os componentes do cerrado, associados a estratégias adaptativas.

\section{Referências Bibliográficas}

ALVIM, P.T. \& W.A. ARAUJO. 1952. El suelo como factor ecológico en el desarollo de la vegetación en el centro oeste del Brasil. Turrialba 2:153-160.

AZEVEDO, L.G. 1967. Tipos eco-físionômicos de vegetação do Território Federal do Amapá. Revta bras. Geogr. 29:25-51.

BATISTA, E.A. 1982. Levantamentos fitossociológicos aplicados à vegetação do cerrado, utilizando-se de fotografias aéreas verticais. Dissertação de Mestrado, Universidade de São Paulo, Piracicaba.

BATISTA, E.A. 1988. Influência de fatores edáficos no cerrado da Reserva Biológica de Moji-Guaçu, SP. Tese de Doutorado. Universidade de São Paulo, Piracicaba.

BORGONOVI, M. \& J.V. CHIARINI. 1965. Cobertura vegetal do Estado de São Paulo. I. Levantamento por fotointerpretaçäo das áreas cobertas com cerrado, cerradão e campo. Bragantia 24:159-172.

CAMARGO, A.P.; R.R. ALFONSI; H.S. PINTO; J.V. CHIARINI. 1976. Zoneamento da aptidão climática para culturas comerciais em áreas de cerrado. In: $4^{2}$ Simpósio sobre o cerrado. (M.G.Ferri, coord.). Itatiaia, Belo Horizonte e EDUSP, São Paulo, 89-120. 
COUTINHO, L.M. 1980. 0 conceito do cerrado. Revta bras. Bot. 1:17-24.

DE VUONO, Y.S.; L.M. BARBOSA; E.A. BATISTA. 1982. A Reserva Biológica de Mogi Guaçu. Silvic. S. Paulo 16A:548-558.

DE VUONO, Y.S.; E.A. BATISTA; F.L. FUNARI. 1986. Balanço hídrico da Reserva Biológica de Mogi Guaçu, São Paulo. Hoehnea 13:73-85.

DONZELLI, J.L.; A. PEREZ Fº; J.F.LEPDCH /; J.B. OLIVEIRA. Levantamento detalhado dos solos da Estação Experimental de Moji Guaçu, SP. (inédito).

DUCKE, A. \& G.A. BLACK. 1954. Phytogeographical notes on the Brazilian amazon. Bolm. téc. Inst. agron. N. 29:1-62.

EITEN, G. 1963. Habitat flora of Fazenda Campininha, São Paulo, Brazil. In: Simpósio sobre o cerrado. (M.G.Ferri, coord.). EDUSP, São Paulo, 179-231.

EITEN, G. 1970. A vegetação do Estado de São Paulo. Bolm Inst. Bot. S. Paulo7:1-147.

EITEN, G. 1972. The cerrado vegetation of Brazil. Bot. Rev. 38:201-341.

FERRI, M.G. 1960. Nota preliminar sobre a vegetação de cerrado em Campo de Mourão (PR). Bolm Fac. Fil. Ciênc. Univ. S. Paulo 51 (Botânica) 4:161-224.

FERRI, M.G. 1977. Ecologia dos cerrados. In $4^{2}$ Simpósio sobre o cerrado. (M.G. Ferri, coord.). Itatiaia, Belo Horizonte, EDUSP, São Paulo, 15-36.

GIBBS, P.E.; H.F. LEITÃO F'; G.J. SHEPHERD. 1983. Floristic composition and community structure in an area of cerrado in SE Brazil. Flora 173:433-449.

GOODLAND, R. 1969. Análise ecológica da vegetação do cerrado. In: Ecologia do cerrado. (R. Goodland \& M.G. Ferri). Itatiaia, Belo Horizonte, EDUSP, São Paulo, 167-179.

HERINGER, E.P.; G.M. BARROSO; J.A. RIZZO; C.T. RIZZINI. 1977. A flora do cerrado. In: $4^{2}$ Simpósio sobre o cerrado. (M.G. Ferri, coord.). Itatiaia, Belo Horizonte, EDUSP, São Paulo, 211-232.

HEYWOOD, V.H. 1978. Flowering plants of the world. Oxford Univ. Press, Oxford. JOLY, A.B. 1977. Botânica: Introdução à taxonomia vegetal. Nacional, São Paulo. LABOURIAU, L.G. 1966. Revisão da situação da ecologia vegetal nos cerrados. Anais Acad. bras. Ciênc. 38 (supl.):5-38.

LAWRENCE, G.H.M. 1973. Taxonomia das plantas vasculares. (M.S.T. Antunes, trad.). Fundação Calouste Gulbenkian, Lisboa.

LOEFGREN, A. 1896. Ensaio para uma distribuição dos vegetais nos diversos grupos florísticos no Estado de São Paulo. Bolm Comm. geogr. geol. S. Paulo 11:1-50.

MANTOVANI, W. 1983. Composição e similaridade florística, fenologia e espectro biológico do cerrado da Reserva Biológica de Moji Guaçu, Estado de São Paulo. Dissertação de Mestrado. Universidade Estadual de Campinas, Campinas.

MANTOVANI ,W. 1987. Análise florística e fitossociológica do estrato herbáceosubarbustivo do cerrado na Reserva Biológica de Moji Guaçu e em Itirapina. Tese de Doutorado. Universidade Estadual de Campinas, Campinas.

MANTOVANI, W. 1990. O estrato herbáceo do cerrado na região Sudeste do Brasil. In: $8^{\circ}$ Congresso da Sociedade Botânica de São Paulo (Sociedade Botânica de São Paulo, editora). SBSP, Campinas. (no prelo). 
MANTOVANI, W. \& F.R. MARTINS. 1988. Variações fenológicas das espécies do cerrado da Reserva Biológica de Moji Guaçu, Estado de São Paulo. Revta bras. Bot. 11:101-112.

MANTOVANI, W.; G.F. LEITÄO; F.R. MARTINS. 1985. Chave baseada em caracteres vegetativos para identificação de espécies lenhosas do cerrado da Reserva Biológica de Moji Guaçu, Estado de São Paulo. Hoehnea 12:35-56.

MUELLER-DOMBOIS, D. \& H. ELLENBERG. 1974. Aims and methods of vegetation ecology. John Wiley \& Sons, New York.

PEREZ F', A.; J.L. DONZELLI; I.F. LEPSCH 1980. Relação solos-geomorfologia em várzea do Rio Mogi Guaçu (S). Revta bras. Ciênc. Solo 4:181-187.

PIJ, L. VAN DER 1972. Principles of dispersal in higher plants. Springer-Verlag, Berlim.

RATTER, J.A. 1980. Notes on the vegetation of Fazenda Água limpa (Brasilia-DF, Brasil). Royal Botanical Garden, Edinburgh.

RAUNKIAER, C.'1934. The life forms of plants and statistical geography. Clarendon, Oxford.

RIZZINI, C.T. 1963a. A flora do cerrado. In Simpósio sobre o cerrado. (M. G. Ferri, coord.). Edgard Blücher e EDUSP, São Paulo, 125-178.

RIZZINI, C.T. 1963b. Nota prévia sobre a divisão fitogeográfica (florística-sociológica) do Brasil. Revta bras. Geogr. 26:3-64.

RIZZINI, C.T. 1979. Tratado da fitogeografia do Brasil: Aspectos ecológicos, HUCITEC e EDUSP, São Paulo.

RODRIGUES, W.A. 1971. Plantas dos campos de Rio Branco (Território de Roraima). In: $3^{9}$ Simpósio sobre o cerrado. (M.G. Ferri, coord.). Edgard Blücher e EDUSP, São Paulo, 180-193.

STELLFELD, C. 1950. Fitogeografia do Estado do Paraná. Archos Mus. Paran. 7:309-349.

TAKEUCHI, M. 1960. The structure of amazonian vegetation. I. Savanna in northern amazon. J. Fac. Sci. Tokyo Univ. sect. 3, Botany, 7:523-533.

TROPPMAIR, H. 1974. A cobertura vegetal primitiva do Estado de São Paulo baseada em estudos toponímicos, históricos e ecológicos. Ciênc. Cult. 26:240243.

VELOSO, H.P. 1963. Os grandes clímaces do Brasil. III: Consideraçōes gerais sobre a vegetação do Centro-Oeste. Mem. Inst. Osw. Cruz 61:357-370.

VELOSO, H.P. 1964. Os grandes clímaces do Brasil. IV: Consideraçōes gerais sobre a vegetação da Região Nordeste. Mem. Inst. Osw. Cruz 62:203-223.

WARMING, E. 1892. Lagoa Santa, contribuição para a geographia phytobiológica. (A. Loefgren, trad.) In: Lagoa Santa e A vegetação dos cerrados brasileiros. (E. Warming \& M.G. Ferri). Itataia, Belo Horizonte, EDUSP, São Paulo, 1284 . 